hep-th/0106178

\title{
NONABELIAN D-BRANES AND NONCOMMUTATIVE GEOMETRY
}

\author{
ROBERT MYERS \\ Department of Physics, McGill University, 3600 University Street \\ Montréal, Québec, H3A 2T8, Canada
}

\begin{abstract}
We discuss the nonabelian world-volume action which governs the dynamics of $\mathrm{N}$ coincident $\mathrm{D} p$-branes. In this theory, the branes' transverse displacements are described by matrix-valued scalar fields, and so this is a natural physical framework for the appearance of noncommutative geometry. One example is the dielectric effect by which $\mathrm{D} p$-branes may be polarized into a noncommutative geometry by external fields. Another example is the appearance of noncommutative geometries in the description of intersecting D-branes of differing dimensions, such as D-strings ending on a D3- or D5-brane. We also describe the related physics of giant gravitons.
\end{abstract}

The idea that noncommutative geometry should play a role in physical theories is an old one [1, 2]. Suggestions have been made that such noncommutative structure may resolve the ultraviolet divergences of quantum field theories, or appear in the description of spacetime geometry at the Planck scale. In the past few years, it has also become a topic of increasing interest to string theorists. From one point of view, the essential step in realizing a noncommutative geometry is replacing the spacetime coordinates by noncommuting operators: $x^{\mu} \rightarrow \hat{x}^{\mu}$. In this replacement, however, there remains a great deal of freedom in defining the nontrivial commutation relations which the operators $\hat{x}^{\mu}$ must satisfy. Some explicit choices that have appeared in physical problems are as follows:

(i) Canonical commutation relations:

$$
\left[\hat{x}^{\mu}, \hat{x}^{\nu}\right]=i \theta^{\mu \nu} \quad \theta^{\mu \nu} \in \mathbf{C}
$$

Such algebras have appeared in the Matrix theory description of planar D-branes [3] — for a review, see [4]. This work also stimulated an ongoing investigation by string theorists of noncommutative field theories which arise in the low energy limit of a planar D-brane with a constant B-field flux — see, e.g., [5, 6, 7].

(ii) Quantum space relations:

$$
\hat{x}^{\mu} \hat{x}^{\nu}=q^{-1} R_{\rho \tau}^{\mu \nu} \hat{x}^{\rho} \hat{x}^{\tau} \quad R_{\rho \tau}^{\mu \nu} \in \mathbf{C}
$$

These algebras received some attention from physicists in the early 1990's — see, e.g., [8, 9] — and have appeared more recently in the geometry of the moduli space of $N=4$ super-Yang-Mills theory [10].

(iii) Lie algebra relations:

$$
\left[\hat{x}^{\mu}, \hat{x}^{\nu}\right]=i f_{\rho}^{\mu \nu} \hat{x}^{\rho} \quad f_{\rho}^{\mu \nu} \in \mathbf{C}
$$


Such algebras naturally arise in the description of fuzzy spheres as was discovered in early attempts to quantize the supermembrane [11, 12]. These noncommutative geometries have also been applied in Matrix theory to describe spherical D-branes [13, 14]. In string theory, these noncommutative descriptions of spheres also arise in various contexts in the physics of D-branes, as will be discussed below.

For a system of $\mathrm{N}$ (nearly) coincident D-branes, the transverse displacements are described by a set of scalar fields, which are matrix-valued in the adjoint representation of $U(N)$. Hence, noncommutative geometries with a Lie-algebra structure appear very naturally in the physics of D-branes. The appearance of a nonabelian $\mathrm{U}(\mathrm{N})$ gauge symmetry in the world-volume theory of $\mathrm{N}$ coincident D-branes [15] is, of course, one of the most remarkable aspects of the D-brane story [16, 17]. It lies at the heart of such recent developments as the entropy counting of nearBPS black holes [18] and the AdS/CFT correspondence [19]. Progress has recently been made on constructing the world-volume action that controls the dynamics of this nonabelian theory [20, 21]. In particular, one finds that this action includes a wide variety of new nonderivative terms for the world-volume scalars. Amongst these interactions are couplings by which the nonabelian D-branes can interact with all of the Ramond-Ramond potentials of any form degree. Further, there is an interesting "dielectric effect" [20] in which the D-branes are polarized into a higher dimensional noncommutative geometry by nontrivial background fields.

An outline of this paper is as follows: We begin in section 1 with a discussion of the nonabelian D-brane action. Section 2 presents an outline of the dielectric effect for D-branes. Section 3 describes the related physical effect by which branes carrying momentum expand in $\mathrm{AdS}_{m} \times \mathrm{S}^{n}$ backgrounds, producing giant gravitons. Finally, section 4 gives a discussion of how noncommutative geometries can arise in the description of intersecting branes. Sections 1 and 2 are essentially a summary of the material appearing in ref. [20]. Section 3 describes that in ref. 222 and section 4 describes that for refs. [23] and [24]. We direct the interested reader to these papers for a more detailed presentation of the associated works.

\section{Nonabelian D-brane action}

Within the framework of perturbative string theory, a $\mathrm{D} p$-brane is a $(p+1)$-dimensional extended surface in spacetime which supports the endpoints of open strings [16, [17]. The massless modes of this open string theory form a supersymmetric U(1) gauge theory with a vector $A_{a}, 9-p$ real scalars $\Phi^{i}$ and their superpartner fermions - for the most part, the latter are ignored throughout the following discussion. At leading order, the low-energy action corresponds to the dimensional reduction of that for ten-dimensional U(1) super-Yang-Mills theory. However, as usual in string theory, there are higher order $\alpha^{\prime}=\ell_{s}^{2}$ corrections - $\ell_{s}$ is the string length scale. For constant field strengths, these stringy corrections can be resummed to all orders, and the resulting action takes the Born-Infeld form [25]

$$
S_{B I}=-T_{p} \int d^{p+1} \sigma\left(e^{-\phi} \sqrt{-\operatorname{det}\left(P[G+B]_{a b}+\lambda F_{a b}\right)}\right)
$$

where $T_{p}$ is the $\mathrm{D} p$-brane tension and $\lambda$ denotes the inverse of the (fundamental) string tension, i.e., $\lambda=2 \pi \ell_{s}^{2}$. This Born-Infeld action describes the couplings of the $\mathrm{D} p$-brane to the massless Neveu-Schwarz fields of the bulk closed string theory, i.e., the metric, dilaton and Kalb-Ramond two-form. The interactions with the massless Ramond-Ramond (RR) fields are incorporated in a second part of the action, the Wess-Zumino term 26, 27, 28

$$
S_{W Z}=\mu_{p} \int P\left[\sum C^{(n)} e^{B}\right] e^{\lambda F}
$$


where $C^{(n)}$ denote the $n$-form RR potentials. Eq. (2) shows that a D $p$-brane is naturally charged under the $(p+1)$-form RR potential with charge $\mu_{p}$, and supersymmetry dictates that $\mu_{p}= \pm T_{p}$. If the $\mathrm{D} p$-brane carries a flux of $B+F$, it will also act as a charge source for RR potentials with a lower form degree [26]. Such configurations represent bound states of D-branes of different dimensions 15.

In both of the expressions above, the symbol $P[\ldots]$ denotes the pull-back of the bulk spacetime tensors to the D-brane world-volume. Thus the Born-Infeld action (11) has a geometric interpretation, i.e., it is essentially the proper volume swept out by the D $p$-brane, which is indicative of the fact that D-branes are actually dynamical objects. This dynamics becomes more evident with an explanation of the static gauge choice implicit in constructing the above action. To begin, we employ spacetime diffeomorphisms to position the world-volume on a fiducial surface defined as $x^{i}=0$ with $i=p+1, \ldots, 9$. With world-volume diffeomorphisms, we then match the world-volume coordinates with the remaining spacetime coordinates on this surface, $\sigma^{a}=x^{a}$ with $a=0,1, \ldots, p$. Now the world-volume scalars $\Phi^{i}$ play the role of describing the transverse displacements of the D-brane, through the identification

$$
x^{i}(\sigma)=2 \pi \ell_{s}^{2} \Phi^{i}(\sigma) \quad \text { with } i=p+1, \ldots, 9 .
$$

With this identification the general formula for the pull-back reduces to

$$
\begin{aligned}
P[E]_{a b} & =E_{\mu \nu} \frac{\partial x^{\mu}}{\partial \sigma^{a}} \frac{\partial x^{\nu}}{\partial \sigma^{b}} \\
& =E_{a b}+\lambda E_{a i} \partial_{b} \Phi^{i}+\lambda E_{i b} \partial_{a} \Phi^{i}+\lambda^{2} E_{i j} \partial_{a} \Phi^{i} \partial_{b} \Phi^{j} .
\end{aligned}
$$

In this way, the expected kinetic terms for the scalars emerge to leading order in an expansion of the Born-Infeld action (11). Note that our conventions are such that both the gauge fields and world-volume scalars have the dimensions of $l e n g t h^{-1}$ — hence the appearance of the string scale in eq. (3).

As N parallel D-branes approach each other, the ground state modes of strings stretching between the different D-branes become massless. These extra massless states carry the appropriate charges to fill out representations under a $U(N)$ symmetry. Hence the $U(1)^{N}$ of the individual D-branes is enhanced to the nonabelian group $\mathrm{U}(\mathrm{N})$ for the coincident D-branes [15]. The vector $A_{a}$ becomes a nonabelian gauge field

$$
A_{a}=A_{a}^{(n)} T_{n}, \quad F_{a b}=\partial_{a} A_{b}-\partial_{b} A_{a}+i\left[A_{a}, A_{b}\right]
$$

where $T_{n}$ are $\mathrm{N}^{2}$ hermitian generators with $\operatorname{Tr}\left(T_{n} T_{m}\right)=\mathrm{N} \delta_{n m}$. The scalars $\Phi^{i}$ also transform in the adjoint of $\mathrm{U}(\mathrm{N})$ with covariant derivatives

$$
D_{a} \Phi^{i}=\partial_{a} \Phi^{i}+i\left[A_{a}, \Phi^{i}\right]
$$

Understanding how to accommodate this $\mathrm{U}(\mathrm{N})$ gauge symmetry in the world-volume action is an interesting puzzle. For example, the geometric meaning or even the validity of eq. (3) seems uncertain when the scalars on the right hand side are matrix-valued. In fact, this identification does remain roughly correct. Some intuition comes from the case where the scalars are commuting matrices and the gauge symmetry can be used to simultaneously diagonalize all of them. In this case, one interpretes the $\mathrm{N}$ eigenvalues of the diagonal $\Phi^{i}$ as representing the 
displacements of the $\mathrm{N}$ constituent D-branes — see, e.g., [4]. Of course, to describe noncommutative geometries, we will be more interested in the case where the scalars do not commute and so cannot be simultaneously diagonlized.

Refs. [20] and [21] recently made progress in constructing the world-volume action describing the dynamics of nonabelian D-branes. The essential strategy in both of these papers was to construct an action which was consistent with the familiar string theory symmetry of T-duality [29]. Acting on D-branes, T-duality acts to change the dimension of the world-volume [16, 17]. The two possibilities are: ( $i$ ) if a coordinate transverse to the $\mathrm{D} p$-brane, e.g., $y=x^{p+1}$, is T-dualized, it becomes a $\mathrm{D}(p+1)$-brane where $y$ is now the extra world-volume direction; and (ii) if a world-volume coordinate on the $\mathrm{D} p$-brane, e.g., $y=x^{p}$, is T-dualized, it becomes a $\mathrm{D}(p-1)$-brane where $y$ is now an extra transverse direction. Under these transformations, the role of the corresponding world-volume fields change as

$$
\text { (i) } \Phi^{p+1} \rightarrow A_{p+1}, \quad(i i) A_{p} \rightarrow \Phi^{p}
$$

while the remaining components of $A$ and scalars $\Phi$ are left unchanged. Hence in constructing the nonabelian action, one can begin with the D9-brane theory, which contains no scalars since the world-volume fills the entire spacetime. In this case, the nonabelian extension of eqs. (1) and (2) is given by simply introducing an overall trace over gauge indices of the nonabelian field strengths appearing in the action [30]. Then applying T-duality transformations on $9-p$ directions yields the nonabelian action for a $\mathrm{D} p$-brane. Of course, in this construction, one also T-dualizes the background supergravity fields according to the known transformation rules [29, 31, 32, 33]. As in the abelian theory, the result for nonabelian action has two distinct pieces [20, 21]: the Born-Infeld term

$$
\begin{aligned}
S_{B I}=-T_{p} \int d^{p+1} & \sigma \operatorname{STr}\left(e^{-\phi} \sqrt{\operatorname{det}\left(Q^{i}{ }_{j}\right)}\right. \\
\times & \left.\sqrt{-\operatorname{det}\left(P\left[E_{a b}+E_{a i}\left(Q^{-1}-\delta\right)^{i j} E_{j b}\right]+\lambda F_{a b}\right)}\right),
\end{aligned}
$$

with $E_{\mu \nu}=G_{\mu \nu}+B_{\mu \nu}$ and $Q^{i}{ }_{j} \equiv \delta^{i}{ }_{j}+i \lambda\left[\Phi^{i}, \Phi^{k}\right] E_{k j} ;$ and the Wess-Zumino term

$$
S_{W Z}=\mu_{p} \int \mathrm{S} \operatorname{Tr}\left(P\left[e^{i \lambda \mathrm{i}_{\Phi} \mathrm{i}_{\Phi}}\left(\sum C^{(n)} e^{B}\right)\right] e^{\lambda F}\right)
$$

Let us enumerate the nonabelian features of this action:

1. Nonabelian field strength: The $F_{a b}$ appearing explicitly in both terms is now nonabelian, of course.

2. Nonabelian Taylor expansion: The bulk supergravity fields are in general functions of all of the spacetime coordinates, and so in the action (8,99), they are implicitly functionals of the nonabelian scalars. For example, the metric functional appearing in the D-brane action would be given by a nonabelian Taylor expansion

$$
\begin{aligned}
G_{\mu \nu} & =\left.\exp \left[\lambda \Phi^{i} \partial_{x^{i}}\right] G_{\mu \nu}^{0}\left(\sigma^{a}, x^{i}\right)\right|_{x^{i}=0} \\
& =\left.\sum_{n=0}^{\infty} \frac{\lambda^{n}}{n !} \Phi^{i_{1}} \cdots \Phi^{i_{n}}\left(\partial_{x^{i_{1}}} \cdots \partial_{x^{i_{n}}}\right) G_{\mu \nu}^{0}\left(\sigma^{a}, x^{i}\right)\right|_{x^{i}=0} .
\end{aligned}
$$


3. Nonabelian Pullback: As was noted in refs. [34, 35], the pullback of various spacetime tensors to the world-volume must now involve covariant derivatives of the nonabelian scalars in order to be consistent with the $\mathrm{U}(\mathrm{N})$ gauge symmetry. Hence eq. (身) is replaced by

$$
P[E]_{a b}=E_{a b}+\lambda E_{a i} D_{b} \Phi^{i}+\lambda E_{i b} D_{a} \Phi^{i}+\lambda^{2} E_{i j} D_{a} \Phi^{i} D_{b} \Phi^{j} .
$$

4. Nonabelian Interior Product: In the Wess-Zumino term (9), $\mathrm{i}_{\Phi}$ denotes the interior product with $\Phi^{i}$ regarded as a vector in the transverse space, e.g., acting on an $n$-form $C^{(n)}=$ $\frac{1}{n !} C_{\mu_{1} \cdots \mu_{n}}^{(n)} d x^{\mu_{1}} \cdots d x^{\mu_{n}}$, we have

$$
\mathrm{i}_{\Phi} \mathrm{i}_{\Phi} C^{(n)}=\frac{1}{2(n-2) !}\left[\Phi^{i}, \Phi^{j}\right] C_{j i \mu_{3} \cdots \mu_{n}}^{(n)} d x^{\mu_{3}} \cdots d x^{\mu_{n}} .
$$

Note that acting on forms, the interior product is an anticommuting operator and hence for an ordinary vector (i.e., a vector $v^{i}$ with values in $\left.\mathbb{R}^{9-p}\right): \mathrm{i}_{v} \mathrm{i}_{v} C^{(n)}=0$. It is only because the scalars $\Phi$ are matrix-valued that eq. (12) yields a nontrivial result.

5. Nonabelian Gauge Trace: As is evident above, both parts of the action are highly nonlinear functionals of the nonabelian fields, and so eqs. (8) and (9) would be incomplete without a precise definition for the ordering of these fields under the gauge trace. Above, STr denotes a maximally symmetric trace. To be precise, the trace includes a symmetric average over all orderings of $F_{a b}, D_{a} \Phi^{i},\left[\Phi^{i}, \Phi^{j}\right]$ and the individual $\Phi^{k}$ appearing in the nonabelian Taylor expansions of the background fields. This choice matches that inferred from Matrix theory [36], and a similar symmetrization arises in the leading order analysis of the boundary $\beta$ functions [35]. Finally we should note that with this definition an expansion of the Born-Infeld term (11) does agree with the string theory to fourth order in $F$ [37, 38], however, it does not seem to capture the full physics of the nonabelian fields in the infrared limit [39. Rather at sixth order, additional terms involving commutators of field strengths must be added to the action 40.

Some other general comments on the nonabelian action are as follows: In the Born-Infeld term (8), there are now two determinant factors as compared to one in the abelian action (11). The second determinant in eq. (\$) is a slightly modified version of that in eq. (国). One might think of this as the kinetic factor, since to leading order in the low energy expansion, it yields the familiar kinetic terms for the gauge field and scalars. In the same way, one can think of the new first factor as the potential factor, since to leading order in the low energy expansion, it reproduces the nonabelian scalar potential expected for the super-Yang-Mills theory — see below. Further note that the first factor reduces to simply one when the scalar fields are commuting, even for general background fields.

As mentioned below eq. (2), an individual $\mathrm{D} p$-brane couples not only to the RR potential with form degree $n=p+1$, but also to the RR potentials with $n=p-1, p-3, \ldots$ through the exponentials of $B$ and $F$ appearing in the Wess-Zumino action (2). Above in eq. (9), $\mathrm{i}_{\Phi} \mathrm{i}_{\Phi}$ is an operator of form degree -2 , and so world-volume interactions appear in the nonabelian action (9) involving the higher RR forms. Hence in the nonabelian theory, a Dp-brane can also couple to the RR potentials with $n=p+3, p+5, \ldots$ through the additional commutator interactions. To make these couplings more explicit, consider the D0-brane action (for which $F$ vanishes):

$$
\begin{array}{r}
S_{C S}=\mu_{0} \int \mathrm{S} \operatorname{Tr}\left(P \left[C^{(1)}+i \lambda \mathrm{i}_{\Phi} \mathrm{i}_{\Phi}\left(C^{(3)}+C^{(1)} B\right)\right.\right. \\
-\frac{\lambda^{2}}{2}\left(\mathrm{i}_{\Phi} \mathrm{i}_{\Phi}\right)^{2}\left(C^{(5)}+C^{(3)} B+\frac{1}{2} C^{(1)} B^{2}\right)
\end{array}
$$




$$
\begin{aligned}
& -i \frac{\lambda^{3}}{6}\left(\mathrm{i}_{\Phi} \mathrm{i}_{\Phi}\right)^{3}\left(C^{(7)}+C^{(5)} B+\frac{1}{2} C^{(3)} B^{2}+\frac{1}{6} C^{(1)} B^{3}\right) \\
& \left.\left.+\frac{\lambda^{4}}{24}\left(\mathrm{i}_{\Phi} \mathrm{i}_{\Phi}\right)^{4}\left(C^{(9)}+C^{(7)} B+\frac{1}{2} C^{(5)} B^{2}+\frac{1}{6} C^{(3)} B^{3}+\frac{1}{24} C^{(1)} B^{4}\right)\right]\right) .
\end{aligned}
$$

Of course, these interactions are reminiscent of those appearing in Matrix theory [3, 41]. For example, eq. (13) includes a linear coupling to $C^{(3)}$, which is the potential corresponding to D2-brane charge,

$$
\begin{aligned}
i \lambda \mu_{0} \int & \operatorname{Tr} P\left[\mathrm{i}_{\Phi} \mathrm{i}_{\Phi} C^{(3)}\right] \\
\quad= & i \frac{\lambda}{2} \mu_{0} \int d t \operatorname{Tr}\left(C_{t j k}^{(3)}(\Phi, t)\left[\Phi^{k}, \Phi^{j}\right]+\lambda C_{i j k}^{(3)}(\Phi, t) D_{t} \Phi^{k}\left[\Phi^{k}, \Phi^{j}\right]\right)
\end{aligned}
$$

where we assume that $\sigma^{0}=t$ in static gauge. Note that the first term on the right hand side has the form of a source for D2-brane charge. This is essentially the interaction central to the construction of D2-branes in Matrix theory with the large N limit [3, 41]. Here, however, with finite $\mathrm{N}$, this term would vanish upon taking the trace if $C_{t j k}^{(3)}$ was simply a function of the world-volume coordinate $t$ (since $\left[\Phi^{k}, \Phi^{j}\right] \in \mathrm{SU}(\mathrm{N})$ ). However, in general these three-form components are functionals of $\Phi^{i}$. Hence, while there would be no "monopole" coupling to D2-brane charge, nontrivial expectation values of the scalars can give rise to couplings to an infinite series of higher "multipole" moments.

Finally we add that by the direct examination of string scattering amplitudes using the methods of refs. 42 and 43], one can verify at low orders the form of the nonabelian interactions in eqs. (8) and (9), including the appearance of the new commutator interactions in the nonabelian Wess-Zumino action [44].

\section{Dielectric Branes}

In this section, we wish to consider certain physical effects arising from the new nonabelian interactions in the world-volume action, given by eqs. (8) and (9). To begin, consider the scalar potential for $\mathrm{D} p$-branes in flat space, i.e., $G_{\mu \nu}=\eta_{\mu \nu}$ with all other fields vanishing. In this case, the entire scalar potential originates in the Born-Infeld term (\$) as

$$
V=T_{p} \operatorname{Tr} \sqrt{\operatorname{det}\left(Q^{i}{ }_{j}\right)}=\mathrm{N} T_{p}-\frac{T_{p} \lambda^{2}}{4} \operatorname{Tr}\left(\left[\Phi^{i}, \Phi^{j}\right]\left[\Phi^{i}, \Phi^{j}\right]\right)+\ldots
$$

The commutator-squared term corresponds to the potential for ten-dimensional $\mathrm{U}(\mathrm{N})$ superYang-Mills theory reduced to $p+1$ dimensions. A nontrivial set of extrema of this potential is given by taking the $9-p$ scalars as constant commuting matrices, i.e.,

$$
\left[\Phi^{i}, \Phi^{j}\right]=0
$$

for all $i$ and $j$. Since they are commuting, the $\Phi^{i}$ may be simultaneously diagonalized and as discussed above, the eigenvalues are interpreted as the separated positions of $\mathrm{N}$ fundamental $\mathrm{D} p$-branes in the transverse space. This solution reflects the fact that a system of $\mathrm{N}$ parallel $\mathrm{D} p$ branes is supersymmetric, and so they can sit in static equilibrium with arbitrary separations in the transverse space [16, 17]. 
From the results described in the previous section, it is clear that in going from flat space to general background fields, the scalar potential is modified by new interactions and so one should reconsider the analysis of the extrema. It turns out that this yields an interesting physical effect that is a precise analog for D-branes of the dielectric effect in ordinary electromagnetism. That is when $\mathrm{D} p$-branes are placed in a nontrivial background field for which the $\mathrm{D} p$-branes would normally be regarded as neutral, e.g., nontrivial $F^{(n)}$ with $n>p+2$, new terms will be induced in the scalar potential, and generically one should expect that there will be new extrema beyond those found in flat space, i.e., eq. (16). In particular, there can be nontrivial extrema with noncommuting expectation values of the $\Phi^{i}$, e.g., with $\operatorname{Tr} \Phi^{i}=0$ but $\operatorname{Tr}\left(\Phi^{i}\right)^{2} \neq$ 0 . This would correspond to the external fields "polarizing" the $\mathrm{D} p$-branes to expand into a (higher dimensional) noncommutative world-volume geometry. This is the analog of the familiar electromagnetic process where an external field may induce a separation of charges in neutral materials. In this case, the polarized material will then carry an electric dipole (and possibly higher multipoles). The latter is also seen in the D-brane analog. When the worldvolume theory is at a noncommutative extremum, the gauge traces of products of scalars will be nonvanishing in various interactions involving the supergravity fields. Hence at such an extremum, the $\mathrm{D} p$-branes act as sources for the latter bulk fields.

To make these ideas explicit, we will now illustrate the process with a simple example. We consider N D0-branes in a constant background RR field $F^{(4)}$, i.e., the field strength associated with D2-brane charge. We find that the D0-branes expand into a noncommutative two-sphere which represents a spherical bound state of a D2-brane and N D0-branes.

Consider a background where only RR four-form field strength is nonvanishing with

$$
F_{t i j k}^{(4)}=-2 f \varepsilon_{i j k} \quad \text { for } i, j, k \in\{1,2,3\}
$$

with $f$ a constant (of dimensions length $h^{-1}$ ). Since $F^{(4)}=d C^{(3)}$, we must consider the coupling of the D0-branes to the RR three-form potential, which is given above in eq. (14). If one explicitly introduces the nonabelian Taylor expansion (10), one finds the leading order interaction may be written as

$$
\frac{i}{3} \lambda^{2} \mu_{0} \int d t \operatorname{Tr}\left(\Phi^{i} \Phi^{j} \Phi^{k}\right) F_{t i j k}^{(4)}(t) .
$$

This final form might have been anticipated since one should expect that the world-volume potential can only depend on gauge invariant expressions of the background field. Given that we are considering a constant background $F^{(4)}$, the higher order terms implicit in eq. (14) will vanish as they can only involve spacetime derivatives of the four-form field strength. Combining eq. (18) with the leading order Born-Infeld potential (15) yields the scalar potential of interest for the present problem

$$
V(\Phi)=\mathrm{N} T_{0}-\frac{\lambda^{2} T_{0}}{4} \operatorname{Tr}\left(\left[\Phi^{i}, \Phi^{j}\right]^{2}\right)-\frac{i}{3} \lambda^{2} \mu_{0} \operatorname{Tr}\left(\Phi^{i} \Phi^{j} \Phi^{k}\right) F_{t i j k}^{(4)}(t)
$$

Substituting in the background field (17) and $\mu_{0}=T_{0}, \delta V(\Phi) / \delta \Phi^{i}=0$ yields

$$
0=\left[\left[\Phi^{i}, \Phi^{j}\right], \Phi^{j}\right]+i f \varepsilon_{i j k}\left[\Phi^{j}, \Phi^{k}\right] .
$$

Note that commuting matrices (16) describing separated D0-branes still solve this equation. The value of the potential for these solutions is simply $V_{0}=\mathrm{N} T_{0}$, the mass of $\mathrm{N}$ D0-branes. Another interesting solution of eq. (20) is

$$
\Phi^{i}=\frac{f}{2} \alpha^{i}
$$


where $\alpha^{i}$ are any $\mathrm{N} \times \mathrm{N}$ matrix representation of the $\mathrm{SU}(2)$ algebra

$$
\left[\alpha^{i}, \alpha^{j}\right]=2 i \varepsilon_{i j k} \alpha^{k}
$$

For the moment, let us focus on the irreducible representation for which one finds

$$
\operatorname{Tr}\left[\left(\alpha_{\mathrm{N}}^{i}\right)^{2}\right]=\frac{\mathrm{N}}{3}\left(\mathrm{~N}^{2}-1\right) \quad \text { for } i=1,2,3 .
$$

Now evaluating the value of the potential (19) for this new solution yields

$$
V_{\mathrm{N}}=\mathrm{N} T_{0}-\frac{T_{0} \lambda^{2} f^{2}}{6} \sum_{i=1}^{3} \operatorname{Tr}\left[\left(\Phi^{i}\right)^{2}\right]=\mathrm{N} T_{0}-\frac{\pi^{2} \ell_{s}^{3} f^{4}}{6 g} \mathrm{~N}^{3}\left(1-\frac{1}{\mathrm{~N}^{2}}\right)
$$

using $T_{0}=1 /\left(g \ell_{s}\right)$. Hence the noncommutative solution (21) has lower energy than a solution of commuting matrices, and so the latter configuration of separated D0-branes is unstable towards condensing out into this noncommutative solution. One can also consider reducible representations of the $\mathrm{SU}(2)$ algebra (22), however, one finds that the corresponding energy is always larger than that in eq. (24). Hence it seems that the irreducible representation describes the ground state of the system.

Geometrically, one can recognize the $\mathrm{SU}(2)$ algebra as that corresponding to the noncommutative or fuzzy two-sphere [12, 15]. The physical size of the fuzzy two-sphere is given by

$$
R=\lambda\left(\sum_{i=1}^{3} \operatorname{Tr}\left[\left(\Phi^{i}\right)^{2}\right] / \mathrm{N}\right)^{1 / 2}=\pi \ell_{s}^{2} f \mathrm{~N}\left(1-\frac{1}{\mathrm{~N}^{2}}\right)^{1 / 2}
$$

in the ground state solution. From the Matrix theory construction of Kabat and Taylor [14], one can infer this ground state is not simply a spherical arrangement of D0-branes rather the noncommutative solution actually represents a spherical D2-brane with N D0-branes bound to it. In the present context, the latter can be verified by seeing that this configuration has a "dipole" coupling to the RR four-form. The precise form of this coupling is calculated by substituting the noncommutative scalar solution (21) into the world-volume interaction (18), which yields

$$
-\frac{R^{3}}{3 \pi g \ell_{s}^{3}}\left(1-\frac{1}{\mathrm{~N}^{2}}\right)^{-1 / 2} \int d t F_{t 123}^{(4)} \text {. }
$$

for the ground state solution. Physically this $F^{(4)}$-dipole moment arises because antipodal surface elements on the sphere have the opposite orientation and so form small pairs of separated membranes and anti-membranes. Of course, the spherical configuration carries no net D2-brane charge.

Given that the noncommutative ground state solution corresponds to a bound state of a spherical D2-brane and N D0-branes, one might attempt to match the above results using the dual formulation. That is, this system can be analyzed from the point of view of the (abelian) world-volume theory of a D2-brane. In this case, one would consider a spherical D2-brane carrying a flux of the $\mathrm{U}(1)$ gauge field strength representing the $\mathrm{N}$ bound D0-branes, and at the same time, sitting in the background of the constant RR four-form field strength (17). In fact, one does find stable static solutions, but what is more surprising is how well the results match those calculated in the framework of the D0-branes. The results for the energy, radius 
and dipole coupling are the same as in eqs. (24), (25) and (26), respectively, except that the factors of $\left(1-1 / \mathrm{N}^{2}\right)$ are absent [20]. Hence for large $\mathrm{N}$, the two calculations agree up to $1 / \mathrm{N}^{2}$ corrections.

One expects that the D2-brane calculations would be valid when $R \gg \ell_{s}$ while naively the D0-brane calculations would be valid when $R \ll \ell_{s}$. Hence it appears there is no common domain where the two pictures can both produce reliable results. However, a more careful consideration of range of validity of the D0-brane calculations only requires that $R \ll \sqrt{\mathrm{N}} \ell_{s}$. This estimate is found by requiring that the scalar field commutators appearing in the full nonabelian potential (15) are small so that the Taylor expansion of the square root converges rapidly. Hence for large $\mathrm{N}$, there is a large domain of overlap where both of the dual pictures are reliable. Note the density of D0-branes on the two-sphere is $\mathrm{N} /\left(4 \pi R^{2}\right)$. However, even if $R$ is macroscopic it is still bounded by $R \ll \sqrt{\mathrm{N}} \ell_{s}$ and so this density must be large compared to the string scale, i.e., the density is much larger than $1 / \ell_{s}^{2}$. With such large densities, one can imagine the discreteness of the fuzzy sphere is essentially lost and so there is good agreement with the continuum sphere of the D2-brane picture.

Finally note that the Born-Infeld action contains couplings to the Neveu-Schwarz two-form which are similar to that in eq. (18). From the expansion of $\sqrt{\operatorname{det}(Q)}$, one finds a cubic interaction

$$
\frac{i}{3} \lambda^{2} T_{0} \int d t \operatorname{Tr}\left(\Phi^{i} \Phi^{j} \Phi^{k}\right) H_{i j k}(t)
$$

Hence the noncommutative ground state, which has $\operatorname{Tr}\left(\Phi^{i} \Phi^{j} \Phi^{k}\right) \neq 0$, also acts as a source of the $B$ field with

$$
-\frac{R_{0}^{3}}{3 \pi g \ell_{s}^{3}}\left(1-\frac{1}{\mathrm{~N}^{2}}\right) \int d t H_{123} .
$$

This coupling is perhaps not so surprising given that the noncommutative ground state represents the bound state of a spherical D2-brane and N D0-branes. Explicit supergravity solutions describing D2-D0 bound states with a planar geometry have been found [46, 47], and are known to carry a long-range $H$ field with the same profile as the RR field strength $F^{(4)}$. One can also derive this coupling from the dual D2-brane formulation. Furthermore, we observe that the presence of this coupling (27) means that we would find an analogous dielectric effect if the $\mathrm{N}$ D0-branes were placed in a constant background $H$ field.

The example considered above must be considered simply a toy calculation demonstrating the essential features of the dielectric effect for D-branes. A more complete calculation would require analyzing the D0-branes in a consistent supergravity background. For example, the present case could be extended to consider the asymptotic supergravity fields of a D2-brane, where the RR four-form would be slowly varying but the metric and dilaton fields would also be nontrivial. Alternatively, one can find solutions with a constant background $F^{(4)}$ in M-theory, namely the $\mathrm{AdS}_{4} \times \mathrm{S}^{7}$ and $\mathrm{AdS}_{7} \times \mathrm{S}^{4}$ backgrounds - see, e.g., 48. In lifting the D0-branes to M-theory, they become gravitons carrying momentum in the internal space. Hence the expanded D2-D0 system considered here correspond to the "giant gravitons" of ref. 449. The analog of the D2-D0 bound state in a constant background $F^{(4)}$ corresponds to M2-branes with internal momentum expanding into $\mathrm{AdS}_{4}$ [22, 50], while the that in a constant $H$ field corresponds to the M2-branes expanding on $S^{4}$ [49]. Alternatively, the dielectric effect has been found to play a role in other string theory contexts, for example, in the resolution of certain singularities in the AdS/CFT correspondence [51, or in describing D-branes in the spacetime background corresponding to a WZW model [52, 53]. Further, one can consider 
more sophisticated background field configurations which through the dielectric effect generate more complicated noncommutative geometries [54].

\section{Giant Gravitons}

From the above discussion, it seems that in the M-theory backgrounds of $\mathrm{AdS}_{4} \times \mathrm{S}^{7}$ or $\mathrm{AdS}_{7} \times \mathrm{S}^{4}$, one will find that an M2-brane carrying internal momentum will expand into a stable spherical configuration. While a Matrix theory description of such states in terms of noncommutative geometry is not yet possible, one can instead analyse these configurations in terms of the abelian world-volume theory of the M2-brane. In fact, the spherical M2-branes expanding into $\mathrm{AdS}_{4}$ were actually discovered some time ago [55]. It turns out that M5-branes will expand in a similar way for these backgrounds, and further that expanded D3-branes arise in the type IIB supergravity background $\mathrm{AdS}_{5} \times \mathrm{S}^{5}$. A detailed analysis [22, 49, 50] shows that these expanded branes are BPS states with the quantum numbers of a graviton. In the following, we will discuss the details of the effect for the D3-branes. Most of the discussion applies equally well for the analogous M2- and M5-brane configurations.

The line element for $\mathrm{AdS}_{5} \times \mathrm{S}^{5}$ may be written as:

$$
d s^{2}=-\left(1+\frac{r^{2}}{L^{2}}\right) d t^{2}+\frac{d r^{2}}{1+\frac{r^{2}}{L^{2}}}+r^{2} d \Omega_{3}^{2}+L^{2}\left(d \theta^{2}+\cos ^{2} \theta d \phi^{2}+\sin ^{2} \theta d \widetilde{\Omega}_{3}^{2}\right)
$$

This background also involves a self-dual RR five-form field strength with terms proportional to the volume forms on the two five-dimensional subspaces: $F^{(5)}=\frac{4}{L}\left[\varepsilon\left(\mathrm{AdS}_{5}\right)+\varepsilon\left(\mathrm{S}^{5}\right)\right]$. With the coordinates chosen above, the four-form potential on the the AdS part of the space is

$$
C_{\text {electric }}^{(4)}=-\frac{r^{4}}{L} d t \varepsilon\left(\mathrm{S}^{3}\right)
$$

where $\varepsilon\left(S^{3}\right)$ is the volume form for the three-sphere described by $d \Omega_{3}^{2}$. Similarly, the potential on the $S^{5}$ is

$$
C_{\text {magnetic }}^{(4)}=L^{4} \sin ^{4} \theta d \phi \varepsilon\left(\tilde{\mathrm{S}}^{3}\right)
$$

where $\varepsilon\left(\tilde{S}^{3}\right)$ is the volume form on $d \widetilde{\Omega}_{3}^{2}$. For the D3-brane configurations of interest, the worldvolume action in eqs. (1) and (2) reduces to:

$$
S_{3}=-T_{3} \int d^{4} \sigma \sqrt{-\operatorname{det}(P[G])}+T_{3} \int P\left[C^{(4)}\right] .
$$

Here, the world-volume gauge field has been set to zero, which will be consistent with the full equations of motion.

Following ref. 49], one can find solutions where a D3-brane has expanded on the $\mathrm{S}^{5}$ to a sphere of fixed $\theta$ while it orbits the $S^{5}$ in the $\phi$ direction. Our static gauge choice matches the spatial world-volume coordinates with the angular coordinates on $d \widetilde{\Omega}_{3}^{2}$, and identifies $\sigma^{0}=t$. Now we consider a trial solution of the form: $\theta=$ constant, $r=0$ and $\phi=\phi(t)$. Substituting this ansatz into the world-volume action (32) and integrating over the angular coordinates, yields the following Lagrangian

$$
\mathcal{L}_{3}=\frac{\mathrm{N}}{L}\left[-\sin ^{3} \theta \sqrt{1-L^{2} \cos ^{2} \theta \dot{\phi}^{2}}+L \sin ^{4} \theta \dot{\phi}\right] .
$$


Here we have introduced the (large positive) integer $\mathrm{N}$ which counts the five-form flux on $\mathrm{S}^{5}$. This is also, of course, the rank of the $\mathrm{U}(\mathrm{N})$ gauge group in the dual super-Yang-Mills theory. Introducing the conjugate angular momentum $P_{\phi}=\delta \mathcal{L}_{3} / \delta \dot{\phi}$, we construct the Hamiltonian:

$$
\mathcal{H}_{3}=P_{\phi} \dot{\phi}-\mathcal{L}_{3}=\frac{\mathrm{N}}{L} \sqrt{p^{2}+\tan ^{2} \theta\left(p-\sin ^{2} \theta\right)^{2}}
$$

where $p=P_{\phi} / \mathrm{N}$. Given that the Hamiltonian is independent of $\phi$, the equations of motion will be solved with constant angular momentum (and hence constant $\dot{\phi}$ ). For fixed $p$, eq. (34) can be regarded as the potential that determines the angle $\theta$ for equilibrium. Examining $\mathcal{H}_{3}$ in detail reveals degenerate minima at $\sin \theta=0$ and $\sin ^{2} \theta=p$, and at any of these minima, the energy is $\mathcal{H}_{3}=P_{\phi} / L$. The expanded configurations are then the giant gravitons of ref. [49]. An important observation is that the minima at $\sin ^{2} \theta=p$ only exist for $p \leq 1$. As $p$ grows beyond $p=1$, the minima at $\theta \neq 0$ are lifted above that at $\sin \theta=0$ and then disappear completely if $p>9 / 8$.

The discussion above indicates that one can also consider the possibility of a brane expanding into the AdS part of the spacetime [22, 50]. That is we wish to find solutions where a D3-brane has expanded to a sphere of constant $r$ while it still orbits in the $\phi$ direction on the $\mathrm{S}^{5}$. Choosing static gauge, we again identify $\sigma^{0}=t$ but match the remaining world-volume coordinates with the angular coordinates on $d \Omega_{3}^{2}$. The trial solution is now: $\theta=0, r=$ constant and $\phi=\phi(t)$. Beginning with the same world-volume action (32] [56], one calculates as before and arrives at the following Hamiltonian

$$
\mathcal{H}_{3}=\frac{\mathrm{N}}{L}\left[\sqrt{\left(1+\frac{r^{2}}{L^{2}}\right)\left(p^{2}+\frac{r^{6}}{L^{6}}\right)}-\frac{r^{4}}{L^{4}}\right] .
$$

where as before $p=P_{\phi} / \mathrm{N}$. Examining $\partial \mathcal{H}_{3} / \partial r=0$, one finds minima located at $r=0$ and $(r / L)^{2}=p$. The energy at each of the minima is $\mathcal{H}_{3}=P_{\phi} / L$. In ref. [22], these expanded configurations were denoted as dual giant gravitons. An essential difference from the previous case, however, is that the minima corresponding to expanded branes persist for arbitrarily large $p$.

It is interesting to consider the motion of these expanded brane configurations. Evaluating $\dot{\phi}$ for any of the above solutions, remarkably one finds the same result: $\dot{\phi}=1 / L$, independent of $P_{\phi}$. Further the center of mass motion for any of the equilibrium configurations in the full tendimensional background is along a null trajectory. For example, for the D3-branes expanded on $\mathrm{S}^{5}$

$$
d s^{2}=-\left(1-L^{2} \cos ^{2} \theta \dot{\phi}^{2}\right) d t^{2}=0
$$

when evaluated for $\dot{\phi}=1 / L$ and $\theta=0$ (= the center of mass position). This is, of course, the expected result for a massless 'point-like' graviton, but it applies equally well for both of the expanded brane configurations. However, note that in the expanded configurations, the motion of each element of the sphere is along a time-like trajectory.

From the point of view of five-dimensional supergravity in the AdS space, the stable brane configurations correspond to massive states with $M=P_{\phi} / L$. Their angular momentum means that these states are also charged under a $\mathrm{U}(1)$ subgroup of the $\mathrm{SO}(6)$ gauge symmetry in the reduced supergravity theory. With the appropriate normalizations, the charge is $Q=P_{\phi} / L$, and hence one finds that these configurations satisfy the appropriate BPS bound [49]. One 
can therefore anticipate that all of these configurations should be supersymmetric. The latter result has been verified by an explicit analysis of the residual supersymmetries [22, 50].

The $\mathrm{AdS}_{5} \times \mathrm{S}^{5}$ background is a maximally supersymmetric solution of the type IIB supergravity equations with 32 residual supersymmetries. That is the background fields are invariant under supersymmetries parameterized by 32 independent Killing spinors. These Killing spinors are determined by setting

$$
\delta \Psi_{M}=D_{M} \epsilon-\frac{i}{480} \Gamma_{M}^{P Q R S T} F_{P Q R S T}^{(5)} \epsilon=0
$$

as the variations of all of the other type IIB supergravity fields vanish automatically. The solutions take the form $\epsilon=M\left(x^{\mu}\right) \epsilon_{0}$ where $\epsilon_{0}$ is an arbitrary constant complex Weyl spinor.

A supersymmetric extension of the abelian world-volume action has been constructed for D3-branes (and all other Dp-branes) in a general supergravity background [57, 58]. This action can be viewed as a four-dimensional nonlinear sigma model with a curved superspace as the target space. Hence the theory is naturally invariant under the target-space supersymmetry. Further however, formulating the action with manifest ten-dimensional Lorentz invariance, requires an additional fermionic invariance on the world-volume called $\kappa$-symmetry. For a test brane configuration where both the target space and world-volume fermions vanish, residual supersymmetries may arise provided there are Killing spinors which satisfy a combined targetspace supersymmetry and $\kappa$-symmetry transformation. The latter amounts to imposing a constraint $\Gamma \epsilon=\epsilon$ where

$$
\Gamma=-\frac{i}{4 !} \varepsilon^{i_{1} \cdots i_{4}} \partial_{i_{1}} X^{M_{1}} \cdots \partial_{i_{4}} X^{M_{4}} \Gamma_{M_{1} \cdots M_{4}}
$$

Of course, this constraint is only evaluated on the D3-brane world-volume. For all of the minima of the potentials in both eqs. (34) or (35), this constraint reduces to imposing the same projection

$$
\left(\Gamma^{t \phi}+1\right) \epsilon_{0}=0
$$

Hence not only are the expanded branes and the point-like state all BPS configurations, all of these configurations preserve precisely the same supersymmetries. Note that this projection is what one might have expected for a massless particle moving along the $\phi$ direction, e.g., one can compare to the supersymmetries gravitational waves propagating in flat space [59].

Much of the interest in giant gravitons comes from an intriguing suggestion [49 that they may be related to the 'stringy exclusion principle' 60, 61, 62, 63. The latter arises in the AdS/CFT correspondence [19] where it is easily understood in the conformal field theory. A family of chiral primary operators in the $N=4$ super-Yang-Mills theory terminates at some maximum weight because the $\mathrm{U}(\mathrm{N})$ gauge group has a finite rank. In terms of the dual AdS description, these operators are associated with single particle states carrying angular momentum on the internal five-sphere. So the appearance of an upper bound on the angular momentum seems mysterious from the point of view of the supergravity theory. The suggestion of McGreevy, Susskind and Toumbas [49] is that if the dual single particle states are identified with the giant gravitons, the D3-branes expanded on the $S^{5}$, then the upper bound is produced by the fact that these BPS states only exist for $p \leq 1$. In fact, this exactly reproduces the desired upper bound on the angular momentum: $P_{\phi} \leq \mathrm{N}$.

Unfortunately this interpretation is not entirely clear because rather than a unique candidate for the graviton state, there are three different ones, including the giant gravitons which expand 
on $\mathrm{S}^{5}$, the dual giant gravitons which expand on $\mathrm{AdS}_{5}$, and the point-like states. All of these configurations have the same angular momentum and energy, and preserve precisely the same supersymmetries. Unfortunately the latter two of the candidates display no upper bound on the angular momentum, and so there is some uncertainty about the proposed mechanism for the stringy exclusion principle.

One tentative suggestion [22] is that the exclusion principle may be realized through the quantum mechanical mixing of these different states. One can find instanton configurations describing tunneling between the point-like states and either of the expanded branes [22, 50], but not between the two expanded D3-brane solutions 64. The suggestion is then that this mixing may spontaneously break supersymmetry in the regime $P_{\phi}>\mathrm{N}$ where there are only two potential graviton states.

Ref. [50] has done some interesting calculations in the context of the dual CFT. They seem to be able to identify certain classical field configurations with same properties as the dual giant gravitons. Further these calculations seem to indicate that the minimum corresponding to the point-like graviton is lifted due to strong coupling effects. This then suggests a picture where the D3-branes expanded on $\mathrm{AdS}_{5}$ are dual to coherent states in the $N=4$ super-Yang-Mills theory, and so do not directly correspond to the chiral primary operators considered in the stringy exclusion principle.

\section{Intersecting Branes}

One interesting aspect of the (abelian) Born-Infeld action (11) is that it supports solitonic configurations describing lower-dimensional branes protruding from the original D-brane 65, 66. 67]. For example, in the case of a D3-brane, one finds spike solutions, known as "bions," corresponding to fundamental strings and/or D-strings extending out of the D3-brane. In these configurations, both the world-volume gauge fields and transverse scalar fields are excited. The gauge field corresponds to that of a point charge arising from the end-point of the attached string, i.e., an electric charge for a fundamental string and a magnetic monopole charge for a D-string. The scalar field describes the deformation of the D3-brane geometry caused by attaching the strings. These solutions seem to have a surprisingly wide range of validity, even near the core of the spike where the fields are no longer slowly varying. In fact, one can show that the electric spike corresponding to a fundamental string is a solution of the full string theory equations of motion 68. Further the dynamics of these solutions, as probed through small fluctuations, agrees with the expected string behavior [69, 70, 71, 72]. In part, these remarkable agreements are probably related to the fact that these are supersymmetric configurations.

For the system of N D-strings ending on a D3-brane, there is also a dual description in terms of the nonabelian world-volume theory of the N D-strings. There one finds solutions which have an interpretation, in terms of noncommutative geometry, as describing the D-strings expanding out in a funnel to become an orthogonal D3-brane. In fact, there is an extensive discussion of this system in the literature — see, e.g., [73, 74, 75, 76, 77, 78, 79, 80] - where the emphasis was on the close connection [73] of the D-string equations to the Nahm equations for BPS monopoles [81]. In ref. [23], our emphasis was on the interpretation of these solutions in terms of noncommutative geometry and the remarkable agreement that one finds with the D3-brane spikes in the large $\mathrm{N}$ limit.

For N D-strings in flat space, the dynamics is determined completely by the Born-Infeld 
action (8) which reduces to 20, 38]

$$
S=-T_{1} \int d^{2} \sigma \operatorname{STr} \sqrt{-\operatorname{det}\left(\eta_{a b}+\lambda^{2} \partial_{a} \Phi^{i} Q_{i j}^{-1} \partial_{b} \Phi^{j}\right) \operatorname{det}\left(Q^{i j}\right)}
$$

where

$$
Q^{i j}=\delta^{i j}+i \lambda\left[\Phi^{i}, \Phi^{j}\right]
$$

Implicitly here, the world-volume gauge field has been set to zero, which will be a consistent truncation for the configurations considered below. With the usual choice of static gauge, we set the world-volume coordinates: $\tau=t=x^{0}$ and $\sigma=x^{9}$. For simplicity, one might consider the leading-order (in $\lambda$ ) equations of motion coming from this action:

$$
\partial^{a} \partial_{a} \Phi^{i}=\left[\Phi^{j},\left[\Phi^{j}, \Phi^{i}\right]\right]
$$

Of course, a simple solution of these equations are constant commuting matrices, as in eq. (16). As discussed in the previous section, such a solution describes $\mathrm{N}$ separated parallel D-strings sitting in static equilibrium.

To find a dual description of the bion solutions of the D3-brane theory [65, 66], one needs a static solution which represents the D-strings expanding into a D3-brane. The corresponding geometry would be a long funnel where the cross-section at fixed $\sigma$ has the topology of a twosphere. In this context, the latter cross-section naturally arises as a fuzzy two-sphere [12, 45] if the scalars have values in an $\mathrm{N} \times \mathrm{N}$ matrix representation of the $\mathrm{SU}(2)$ algebra (22). Hence one is lead to consider the ansatz

$$
\Phi^{i}=\frac{R(\sigma)}{\lambda \sqrt{\mathrm{N}^{2}-1}} \alpha^{i}, \quad i=1,2,3,
$$

where we will focus on case where the $\alpha^{i}$ are the irreducible $N \times N \mathrm{SU}(2)$ matrices. Then with the normalization in eq. (43), the function $|R(\sigma)|$ corresponds precisely to the radius of the fuzzy two-sphere

$$
R(\sigma)^{2}=\frac{\lambda^{2}}{\mathrm{~N}} \sum_{i=1}^{3} \operatorname{Tr}\left[\Phi^{i}(\sigma)^{2}\right] .
$$

Substituting the ansatz (43) into the matrix equations of motion (42) yields a single scalar equation

$$
R^{\prime \prime}(\sigma)=\frac{8}{\lambda^{2}\left(\mathrm{~N}^{2}-1\right)} R(\sigma)^{3}
$$

for which one simple class of solutions is

$$
R(\sigma)= \pm \frac{\mathrm{N} \pi \ell_{s}^{2}}{\sigma-\sigma_{\infty}}\left(1-\frac{1}{\mathrm{~N}^{2}}\right)^{1 / 2}
$$

Given the above analysis, eqs. (43) and (46) only represent a solution of the leading order equations of motion (42), and so naively one expects that it should only be valid for small radius. However, one can show by direct evaluation [23] that in fact these configurations solve the full equations of motion extremizing the nonabelian action (40). The latter can also be inferred from an analysis of the world-volume supersymmetry of these configurations. Killing 
spinor solutions of the linearized supersymmetry conditions will exist provided that the scalars satisfy

$$
D_{\sigma} \Phi^{i}= \pm \frac{i}{2} \varepsilon^{i j k}\left[\Phi^{j}, \Phi^{k}\right]
$$

The latter can be recognized as the Nahm equations [73]. Hence the duality between the D3brane and D-string descriptions gives a physical realization of Nahm's transform of the moduli space of BPS magnetic monopoles. Now inserting the ansatz (43) into eq. (47) yields

$$
R^{\prime}=\mp \frac{2}{\lambda \sqrt{N^{2}-1}} R^{2}
$$

which one easily verifies is satisfied by the configuration given in eq. (46). Hence, one concludes that the solutions given by eqs. (43) and (46) are in fact BPS solutions preserving 1/2 of the supersymmetry of the leading order D-string theory. Now in ref. [82], it was shown that BPS solutions of the leading order theory are also BPS solutions of the full nonabelian Born-Infeld action (40).

The geometry of the solution, eqs. (43) and (46), certainly has the desired funnel shape. The fuzzy two-sphere shrinks to zero size as $\sigma \rightarrow \infty$ and opens up to fill the $x^{1,2,3}$ hypersurface at $\sigma=\sigma_{\infty}$. By examining the nonabelian Wess-Zumino action (9), one can show that the noncommutative solution induces a coupling to the RR four-form potential $C_{t 123}^{(4)}$. This calculation confirms then that, with the minus (plus) sign in eq. (46), the D-strings expand into $\mathrm{a}$ (n anti-)D3-brane which fills the $x^{1,2,3}$ directions [23]. Given that the funnel solution of the D-string theory and the bion spike of the D3-brane theory are both BPS, one might expect that there will be a good agreement between these two dual descriptions. The formula for the height of D3-brane spike above the $x^{1,2,3}$ hyperplane is 65

$$
\sigma-\sigma_{\infty}=\frac{\mathrm{N} \pi \ell_{s}^{2}}{R}
$$

Comparing to eq. (46), one finds that for large $\mathrm{N}$ the two descriptions are describing the same geometry up to $1 / \mathrm{N}^{2}$ corrections. One finds similar quantitative agreement for large $\mathrm{N}$ in calculating the energy, the RR couplings and the low energy dynamics in the two dual descriptions [23]. As in the discussion of the dielectric effect, one can argue that the D3-brane description is valid for $R \gg \ell_{s}$ while the D-string description is reliable for $R \ll \sqrt{\mathrm{N}} \ell_{s}[23]$. Hence one can understand the good agreement between these dual approaches for large $\mathrm{N}$ since there is a large domain of overlap where both are reliable.

Note that in the configurations considered in this section, there are no nontrivial supergravity fields in the ambient spacetime. Hence the appearance of the noncommutative geometry in these solutions is quite distinct from that in the dielectric effect, where the external fields drive the D-branes into a certain geometry in the ground state. In the funnel solutions, the noncommutative geometry was put into the ansatz (43) by hand. An interesting extension of these solutions is then to replace the $\mathrm{SU}(2)$ generators by those corresponding to some other noncommutative geometry, i.e., to replace eq. (43) by

$$
\Phi^{i}=\frac{R(\sigma)}{\lambda \sqrt{C}} G^{i}
$$

where the $G^{i}$ are new $\mathrm{N} \times \mathrm{N}$ constant matrices satisfying $\sum\left(G^{i}\right)^{2}=N C$. An interesting feature of such a construction is that near the core of the funnel, the leading order equations of motion 
will still be those given in eq. (42). Thus for eq. (50) to provide a solution, the new generators must satisfy $\left[G^{j},\left[G^{j}, G^{i}\right]\right]=2 a^{2} G^{i}$ for some constant $a$, and then the radius is determined by

$$
R^{\prime \prime}=\frac{2 a^{2}}{\lambda^{2} C} R^{3}
$$

which still has essentially the same form as eq. (45) above. Further the funnel solution of this equation also has essentially the same form as eq. (46) above, i.e.,

$$
R= \pm \frac{\lambda \sqrt{C}}{a\left(\sigma-\sigma_{\infty}\right)} .
$$

Hence the profile with $R \simeq \lambda / \sigma$ is universal for all funnels on the D-string, independent of the details of the noncommutative geometry that describes the cross-section of the funnel.

This universal behavior is curious. For example, one could consider using this framework to describe a D-string ending on an orthogonal $\mathrm{D} p$-brane with $p>3$. However, from the dual $\mathrm{D} p$-brane formulation, one expects that for large $R$, solutions will essentially be harmonic functions behaving like $\sigma \propto R^{-(p-2)}$ or $R \propto \sigma^{-1 /(p-2)}$. The resolution of this puzzle seems to be that the two profiles apply in distinct regimes, the first for small $R$ and the second for large $R$. Hence it must be that the nonlinearity of the full Born-Infeld action will generate solutions which display a transition from one kind of behavior to another.

One particular example that we have examined in detail [24] is the case where $G^{i}$ in eq. (50) are chosen to be generators describing a fuzzy four-sphere - these may be found in, e.g., ref. [14. In this case, the funnel describes the D-strings expanding into a D5-brane. One does find the expected transition in the behavior of the geometry. That is, $\sigma \approx \mathrm{N}^{2 / 3} \ell_{s} / R$ for small $R$ in accord with eq. (52), while at large $R$, higher order terms in the Born-Infeld action (40) become important yielding $\sigma \approx \mathrm{N}^{2 / 3} \ell_{s}^{4} / R^{3}$. The same kind of behavior is also found for the corresponding solutions in the dual D5-brane world-volume theory, although of course in that case the nonlinearities of the Born-Infeld action become important for small $R$. An interesting feature of the D5-brane spike is that it is also nonabelian in character. Charge conservation arguments indicate that the D-string acts as a source of the second Chern class in the world-volume of the D5-brane [83]. More precisely, if N D-strings end on a D5-brane, then

$$
\frac{1}{8 \pi^{2}} \int_{S^{4}} \operatorname{Tr}(F \wedge F)=\mathrm{N}
$$

for any four-sphere surrounding the D-string endpoint. Hence both of the dual descriptions have a noncommutative character. Again, we find that the dual constructions seem to agree at large $\mathrm{N}$, however, the details of the solutions are more complex. In part, the latter must be due to the fact that the $D 5 \perp D 1$ system is not supersymmetric.

\section{Acknowledgments}

This research was supported by NSERC of Canada and Fonds FCAR du Québec. I would like to thank Neil Constable, Marc Grisaru and Øyvind Tafjord for collaborations in the research presented in refs. 222], [23] and [24]. I would also like to thank Neil Constable and Øyvind Tafjord for proofreading a draft of this paper. I would like to thank the organizers of the Workshop on Strings, Duality and Geometry (at Université de Montreal, March 2000) and of Strings 2000 (at University of Michigan, July 2000) for the opportunity to speak on the material discussed in this article. Finally I would like to thank Mike Duff for the invitation to contribute to this special issue of Journal of Mathematical Physics devoted to Strings, Branes and M-theory. 


\section{References}

[1] H.S. Snyder, Quantized Space-Time, Phys. Rev. 71 (1947) 38; The Electromagnetic Field In Quantized Space-Time, Phys. Rev. 72 (1947) 68.

[2] A. Connes, Noncommutative Geometry, Academic Press (1994).

[3] T. Banks, W. Fischler, S.H. Shenker and L. Susskind, $M$ theory as a matrix model: A conjecture, Phys. Rev. D55 (1997) 5112 hep-th/9610043.

[4] W. Taylor, Lectures on D-branes, gauge theory and M(atrices), given at 2nd Trieste Conference on Duality in String Theory, Trieste, Italy, 16-20 Jun 1997 hep-th/9801182; The $M$ (atrix) model of $M$-theory, lectures given at NATO Advanced Study Institute on Quantum Geometry, Akureyri, Iceland, 10-20 Aug 1999 [hep-th/0002016].

[5] A. Connes, M.R. Douglas and A. Schwarz, Noncommutative geometry and matrix theory: Compactification on tori, JHEP 9802 (1998) 3 [hep-th/9711162.

[6] M.R. Douglas and C. Hull, D-branes and the noncommutative torus, JHEP 9802 (1998) 8 hep-th/9711165.

[7] N. Seiberg and E. Witten, String theory and noncommutative geometry, JHEP 9909 (1999) 32 [hep-th/9908142].

[8] J. Wess and B. Zumino, Covariant Differential Calculus on the Quantum Hyperplane, Nucl. Phys. Proc. Suppl. 18B (1991) 302.

[9] P. Schupp, P. Watts and B. Zumino, Differential geometry on linear quantum groups, Lett. Math. Phys. 25 (1992) 139 [hep-th/9206029].

[10] D. Berenstein, V. Jejjala and R.G. Leigh, Marginal and relevant deformations of $N=4$ field theories and non-commutative moduli spaces of vacua, hep-th/0005087; Noncommutative moduli spaces, dielectric tori and $T$ duality, hep-th/0006168.

[11] J. Hoppe, Quantum Theory of a Massless Relativistic Surface and a Two-Dimensional Bound State Problem, MIT Ph.D. thesis, 1982 (available at http://www.aeipotsdam.mpg.de/ hoppd); Elem. Part. Res. J. (Kyoto) 80 (1989) 145.

[12] B. de Wit, J. Hoppe and H. Nicolai, On the quantum mechanics of supermembranes, Nucl. Phys. B305 (1988) 545.

[13] D. Kabat and W. Taylor, Spherical membranes in matrix theory, Adv. Theor. Math. Phys. 2 (1998) 181 [hep-th/9711078.

[14] J. Castelino, S. Lee and W. Taylor, Longitudinal 5-branes as 4-spheres in matrix theory, Nucl. Phys. B526 (1998) 334 [hep-th/9712105].

[15] E. Witten, Bound States Of Strings And p-Branes, Nucl. Phys. B460 (1996) 335 [hepth/9510135.

[16] J. Polchinski, TASI lectures on D-branes, hep-th/9611050. 
[17] C.V. Johnson, D-brane primer, hep-th/0007170.

[18] A.W. Peet, TASI lectures on black holes in string theory, hep-th/0008241.

[19] O. Aharony, S.S. Gubser, J. Maldacena, H. Ooguri and Y. Oz, Large N field theories, string theory and gravity, Phys. Rept. 323 (2000) 183 hep-th/9905111].

[20] R.C. Myers, Dielectric-branes, JHEP 9912 (1999) 22 [hep-th/9910053].

[21] W. Taylor and M. Van Raamsdonk, Multiple Dp-branes in weak background fields, Nucl. Phys. B573 (2000) 703 hep-th/9910052.

[22] M. T. Grisaru, R. C. Myers and Ø. Tafjord, SUSY and Goliath, JHEP 0008 (2000) 040 hep-th/0008015.

[23] N.R. Constable, R.C. Myers and Ø. Tafjord, The noncommutative bion core, Phys. Rev. D61 (2000) 106009 hep-th/9911136.

[24] N.R. Constable, R.C. Myers and Ø. Tafjord, Non-abelian Brane Intersections, hepth/0102080.

[25] R.G. Leigh, Dirac-Born-Infeld Action From Dirichlet Sigma Model, Mod. Phys. Lett. A4 (1989) 2767.

[26] M.R. Douglas, Branes within Branes, hep-th/9512077.

[27] M. Li, Boundary States of D-Branes and Dy-Strings, Nucl. Phys. B460 (1996) 351 [hepth/9510161].

[28] M.B. Green, J.A. Harvey and G. Moore, I-brane inflow and anomalous couplings on Dbranes, Class. Quant. Grav. 14 (1997) 47 [hep-th/9605033.

[29] A. Giveon, M. Porrati and E. Rabinovici, Target space duality in string theory, Phys. Rept. 244 (1994) 77 hep-th/9401139.

[30] M.R. Douglas, D-branes and matrix theory in curved space, Nucl. Phys. Proc. Suppl. 68 (1998) 381 hep-th/9707228]; D-branes in curved space, Adv. Theor. Math. Phys. 1 (1998) 198 [hep-th/9703056].

[31] T.H. Buscher, Quantum Corrections And Extended Supersymmetry In New Sigma Models, Phys. Lett. B159 (1985) 127; A Symmetry Of The String Background Field Equations, Phys. Lett. B194 (1987) 59: Path Integral Derivation Of Quantum Duality In Nonlinear Sigma Models, Phys. Lett. B201 (1988) 466.

[32] E. Bergshoeff, C. Hull and T. Ortin, Duality in the type II superstring effective action, Nucl. Phys. B451 (1995) 547 [hep-th/9504081].

[33] P. Meessen and T. Ortin, An Sl(2,Z) multiplet of nine-dimensional type II supergravity theories, Nucl. Phys. B541 (1999) 195 hep-th/9806120.

[34] C.M. Hull, Matrix theory, U-duality and toroidal compactifications of M-theory, JHEP 9810 (1998) 11 hep-th/9711179. 
[35] H. Dorn, Nonabelian gauge field dynamics on matrix D-branes, Nucl. Phys. B494 (1997) 105 [hep-th/9612120].

[36] W. Taylor and M. Van Raamsdonk, Multiple D0-branes in weakly curved backgrounds, Nucl. Phys. B558 (1999) 63 [hep-th/9904095].

[37] A.A. Tseytlin, On non-abelian generalisation of the Born-Infeld action in string theory, Nucl. Phys. B501 (1997) 41 [hep-th/9701125].

[38] A.A. Tseytlin, Born-Infeld action, supersymmetry and string theory, hep-th/9908105.

[39] A. Hashimoto and W. Taylor, Fluctuation spectra of tilted and intersecting D-branes from the Born-Infeld action, Nucl. Phys. B503 (1997) 193 hep-th/9703217.

[40] P. Bain, On the non-Abelian Born-Infeld action, hep-th/9909154.

[41] T. Banks, N. Seiberg and S. Shenker, Branes from matrices, Nucl. Phys. B490 (1997) 91 hep-th/9612157.

[42] M.R. Garousi and R.C. Myers, Superstring scattering from D-branes, Nucl. Phys. B475 (1996) 193 hep-th/9603194.

[43] A. Hashimoto and I.R. Klebanov, Scattering of strings from D-branes, Nucl. Phys. Proc. Suppl. 55B (1997) 118 hep-th/9611214.

[44] M.R. Garousi and R.C. Myers, World-volume Interactions on D-branes, Nucl. Phys. B542 (1999) 73 [hep-th/9809100]; World-volume Potentials on D-branes, JHEP 0011 (2000) 032 hep-th/0010122.

[45] J. Madore, An Introduction of Noncommutative Differential Geometry and its Applications, Cambridge University Press, Cambridge, 1995.

[46] J.C. Breckenridge, G. Michaud and R.C. Myers, More D-brane bound states, Phys. Rev. D55 (1997) 6438 [hep-th/9611174].

[47] J.G. Russo and A.A. Tseytlin, Waves, boosted branes and BPS states in M-theory, Nucl. Phys. B490 (1997) 121 [hep-th/9611047].

[48] M. J. Duff, TASI lectures on branes, black holes and anti-de Sitter space, hep-th/9912164.

[49] J. McGreevy, L. Susskind and N. Toumbas, Invasion of the giant gravitons from anti-de Sitter space, JHEP 0006 (2000) 008 hep-th/0003075.

[50] A. Hashimoto, S. Hirano and N. Itzhaki, Large branes in AdS and their field theory dual, JHEP 0008 (2000) 051 hep-th/0008016.

[51] J. Polchinski and M.J. Strassler, The string dual of a confining four-dimensional gauge theory, hep-th/0003136.

[52] C. Bachas, M. Douglas and C. Schweigert, Flux stabilization of D-branes, JHEP 0005 (2000) 048 hep-th/0003037. 
[53] M. Li, Fuzzy gravitons from uncertain spacetime, Phys. Rev. D63 (2001) 086002 [hepth/0003173.

[54] S.P. Trivedi and S. Vaidya, Fuzzy cosets and their gravity duals, JHEP 0009 (2000) 041 hep-th/0007011.

[55] E. Bergshoeff, M. J. Duff, C. N. Pope and E. Sezgin, Compactifications Of The ElevenDimensional Supermembrane, Phys. Lett. B224 (1989) 71.

[56] Our conventions are such that we actually consider an anti-D3-brane here [22]. That is the sign of the Wess-Zumino term in eq. (32) was reversed.

[57] M. Cederwall, A. van Gussich, B.E.W. Nilsson and A. Westerberg, The Dirichlet superthree-brane in ten-dimensional type IIB supergravity, Nucl. Phys. B490 (1997) 163 [hepth/9610148; The Dirichlet super-p-branes in ten-dimensional type IIA and IIB supergravity, Nucl. Phys. B490 (1997) 179 [hep-th/9611159].

[58] E. Bergshoeff and P.K. Townsend, Super D-branes, Nucl. Phys. 490 (1997) 145 [hepth/9611173.

[59] E. A. Bergshoeff, R. Kallosh and T. Ortin, Supersymmetric string waves, Phys. Rev. D47 (1993) 5444 hep-th/9212030.

[60] J. Maldacena and A. Strominger, $A d S_{3}$ black holes and a stringy exclusion principle, JHEP 9812 (1998) 005 hep-th/9804085.

[61] A. Jevicki and S. Ramgoolam, Non-commutative gravity from the AdS/CFT correspondence, JHEP 9904 (1999) 032 hep-th/9902059.

[62] P. Ho, S. Ramgoolam and R. Tatar, Quantum spacetimes and finite $N$ effects in $4 D$ superYang-Mills theories, Nucl. Phys. B573 (2000) 364 hep-th/9907145.

[63] S.S. Gubser, Can the effective string see higher partial waves?, Phys. Rev. D56 (1997) 4984 hep-th/9704195.

[64] J. Lee, Tunneling between the giant gravitons in $A d S_{5} \times S^{5}$, hep-th/0010191.

[65] C.G. Callan and J.M. Maldacena, Brane dynamics from the Born-Infeld action, Nucl. Phys. B513 (1998) 198 hep-th/9708147.

[66] G.W. Gibbons, Born-Infeld particles and Dirichlet p-branes, Nucl. Phys. B514 (1998) 603 hep-th/9709027.

[67] P.S. Howe, N.D. Lambert and P.C. West, The self-dual string soliton, Nucl. Phys. B515 (1998) 203 hep-th/9709014.

[68] L. Thorlacius, Born-Infeld string as a boundary conformal field theory, Phys. Rev. Lett. 80 (1998) 1588 hep-th/9710181.

[69] S. Lee, A. Peet and L. Thorlacius, Brane-waves and strings, Nucl. Phys. B514 (1998) 161 hep-th/9710097. 
[70] D. Bak, J. Lee and H. Min, Dynamics of BPS states in the Dirac-Born-Infeld theory, Phys. Rev. D59 (1999) 045011 hep-th/9806149.

[71] K.G. Savvidy and G.K. Savvidy, Von Neumann boundary conditions from Born-Infeld dynamics, Nucl. Phys. B561 (1999) 117 [hep-th/9902023].

[72] D. Kastor and J. Traschen, Dynamics of the DBI spike soliton, Phys. Rev. D61 (2000) 024034 hep-th/9906237.

[73] D.-E. Diaconescu, D-branes, monopoles and Nahm equations, Nucl. Phys. B503 (1997) 220 [hep-th/9608163.

[74] J.P. Gauntlett, J. Gomis, P.K. Townsend, BPS bounds for worldvolume branes, JHEP 9801 (1998) 003 hep-th/9711205.

[75] D. Brecher, BPS states of the non-Abelian Born-Infeld action, Phys. Lett. B442 (1998) 117 [hep-th/9804180].

[76] A. Giveon and D. Kutasov, Brane dynamics and gauge theory, Rev. Mod. Phys. 71 (1999) 983 [hep-th/9802067].

[77] A. Kapustin and S. Sethi, The Higgs branch of impurity theories, Adv. Theor. Math. Phys. 2 (1998) 571 [hep-th/9804027].

[78] D. Tsimpis, Nahm equations and boundary conditions, Phys. Lett. B433 (1998) 287 [hepth/9804081.

[79] K. Hashimoto, String junction from worldsheet gauge theory, Prog. Theor. Phys. 101 (1999) 1353, hep-th/9808185.

[80] A. Gorsky and K. Selivanov, Junctions and the fate of branes in external fields, Nucl. Phys. B571 (2000) 120 [hep-th/9904041].

[81] W. Nahm, A Simple Formalism For The BPS Monopole, Phys. Lett. B90 (1980) 413; The construction of all self-dual multimonopoles by the ADHM method, in Monopoles in quantum field theory, Craigie et al. (eds), World Scientific, Singapore (1982).

[82] A. Hashimoto, The shape of branes pulled by strings, Phys. Rev. D57 (1998) 6441 [hepth/9711097].

[83] G.W. Semenoff and K. Zarembo, Solitons on branes, Nucl. Phys. B556 (1999) 247 [hepth/9903140]. 